\title{
Selective Characterization of Proteins on Nanoscale Concave Surfaces
}

\author{
Xi Qian ${ }^{123}$, Utthara Rameshbabu ${ }^{12}$, Jonathan S. Dordick ${ }^{1234^{*}}$, Richard W. Siegel ${ }^{123^{*}}$
}

1. Department of Materials Science and Engineering, Rensselaer Polytechnic Institute, Troy NY 12180

2. Rensselaer Nanotechnology Center, Rensselaer Polytechnic Institute, Troy, NY 12180

3. Center for Biotechnology and Interdisciplinary Studies, Rensselaer Polytechnic Institute, Troy NY 12180

4. Department of Chemical and Biological Engineering, Rensselaer Polytechnic Institute, Troy NY 12180

*Corresponding authors: Jonathan S. Dordick, email: dordick@rpi.edu, fax: 518-276-2207

Richard W. Siegel, email: rwsiegel@rpi.edu, fax: (518) 276-6540

\begin{abstract}
Nanoscale curvature plays a critical role in nanostructure-biomolecule interactions, yet the understanding of such effects in concave nanostructures is still very limited. Because concave nanostructures usually possess convex surface curvatures as well, it is challenging to selectively study the proteins on concave surfaces alone. In this work, we have developed a novel and facile method to address this issue by desorbing proteins on the external surfaces of hollow gold nanocages (AuNG), allowing the selective characterization of retained proteins immobilized on their internal concave surfaces. The selective desorption of proteins was achieved via varying the solution ionic strength, and was demonstrated by both calculation and experimental comparison with non-hollow nanoparticles. This method has created a new platform for the discrete observation of proteins adsorbed inside AuNG hollow cores, and this work suggests an expanded biomedical application space for hollow nanomaterials.
\end{abstract}

Keywords: gold nanoparticle; concave nanostructure; protein adsorption; nano-bio interaction.
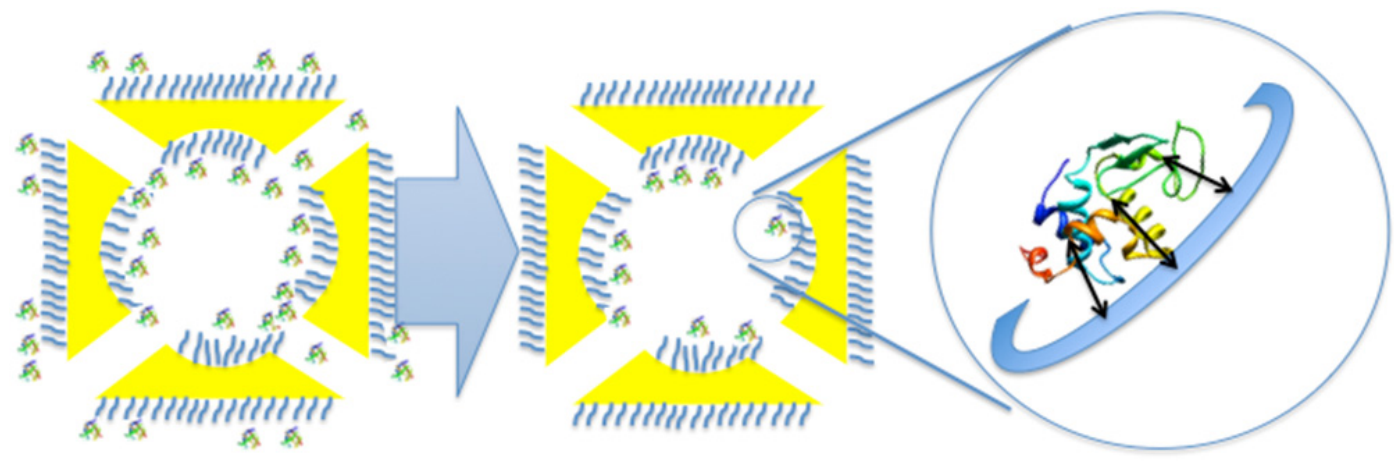


\section{Introduction}

Nanostructured materials have exhibited significant potential for biomedical applications, rendering the investigation of nanostructure-biomolecule (nano-bio) interactions critical regarding both the efficacy and safety of nano-biomaterials[1-4]. In particular, nanoscale morphology has been shown to elicit significant biomolecular responses, as proteins appear to "sense" variations in the topography of their nanoscale environments, and alter their conformation and hence their function[5,6], accordingly. During the last decade, studies on these "morphology effects" have demonstrated the critical nature of surface curvature in affecting biomolecule adsorption, conformation, and activity[7-11]. While the vast majority of these studies have focused on surfaces with positive (convex) curvature, there remains a dearth of systematic studies of the structure and function of biomolecules adsorbed on negatively curved (concave) surfaces. To help address this lack of understanding, galvanically-synthesized gold nanocages (AuNGs) were previously employed by our group to study the effect of concave surfaces[12]. AuNG have hollow cores with concave surfaces[13] and are distinct from other concave nanostructures due to their excellent morphological controllability, stability and protein compatibility[14]. Moreover, due to the surface plasmon coupling effect, additional hotspots can exist inside the hollow core of AuNGs as they aggregate, further facilitating the selective spectroscopic characterization of internally adsorbed macromolecules[15]. In our earlier work, we demonstrated that AuNG provide an excellent platform to study this type of nano-bio interaction. However, it remained a challenge to clearly distinguish the effects on internally and externally adsorbed proteins on AuNG. 
While some experimental methods can, to a limited extent, serve this purpose of distinguishing between internally and externally adsorbed proteins (e.g., additional modification of nanoparticle external surfaces[16] or proteolysis of external proteins[17,18]), a simple procedure to minimize damage to the adsorbed proteins is still desired. In the present study, we employed a simple ionic strength-based method to selectively remove the externally bound proteins on AuNG, taking advantage of hindered through-pore diffusion in AuNG to regulate protein leaching from the internal concave cavities into the highly ionic buffer. Non-hollow gold nanocubes (AuNCs) were used as a reference to verify external protein removal, and surface-enhanced Raman spectroscopy (SERS) was employed to characterize ligand and protein conformation. Lysozyme was chosen in this study to probe the nano-bio interactions, as the enzyme's structure is well characterized and it is known to undergo structural changes upon adsorption to nanoparticle surfaces[19,20]. This combination of methods enabled the specific observation of proteins on nanoscale concave surfaces selectively, without interference from otherwise adsorbed proteins.

\section{Materials and Methods}

\subsection{Materials and instrumentation}

Gold (III) chloride trihydrate (99.9\%), silver nitrate ACS reagent (99+\%), 11-mercaptoundecanoic acid (11-MUA) (90\%), sodium sulfide nonahydrous, 1,5-pentanediol (96\%) (PD), and polyvinylpyrrolidone $\left(\mathrm{M}_{\mathrm{w}} \sim 55000\right)(\mathrm{PVP})$ were purchased from Sigma-Aldrich (St. Louis, MO). Ethylene glycol was purchased from J. T. Baker (Center Valley, PA). Lysozyme (Lyz) from hen egg white, and 4-methylumbelliferyl $\beta$-D-N, N', N"-triacetylchitotrioside 
(4-MU- $\beta(\text { GluNAc) })_{3}$, and Micrococcus lysodeikticus were purchased from Sigma-Aldrich as dry powders and used without further purification. Bicinchoinic acid (BCA) and micro-bicinchoninic acid ( $\mu \mathrm{BCA})$ assay reagents were purchased from Pierce Biotechnology, Inc. (Rockford, IL).

Scanning electron microscopy (SEM) was performed via a Carl Zeiss Supra-55 VP field-emission SEM (Jena, Germany); samples were prepared by drop-drying aqueous nanoparticle suspensions on silicon wafers. Inductively coupled plasma mass spectroscopy (ICP-MS) was performed using a Bruker Varian 820-MS ICP-MS (Billerica, MA), and the samples were prepared by dissolving nanoparticle suspensions into aqua regia and then diluted in 5\% nitric acid. Raman and surface-enhanced Raman spectroscopies (SERS) were performed on a Renishaw Ramascope (Gloucestershire, UK) attached with a Carl-Zeiss optical microscope. The $785 \mathrm{~nm}$ excitation laser was from a semiconductor $\mathrm{cw}$ diode and used with a holographic notch filter and a transmission grating of 1200 lines per millimeter. Drop-dried samples were used to measure nanoparticle ligand conformation, and liquid samples were used to measure protein conformation. In both cases, Raman spectra were collected in backscattered configuration and processed in Wire 3.3 software, also provided by Renishaw. UV-vis spectroscopy measurements were made on a Hitachi U-2910 spectrophotometer.

\subsection{Preparation of nanobioconjugates and removal of externally adsorbed proteins}

AuNG and AuNC nanoparticles were synthesized and surface-modified according to previously described protocols[21,22] with minor modifications[12] and Lyz-nanoparticle conjugates were also prepared as previously described[12]. Briefly, $\sim 100 \mu \mathrm{g} / \mathrm{ml}$ Lyz was incubated 
with $\sim 2 \mathrm{nM}$ AuNG or AuNC in the $2 \mathrm{mM}, \mathrm{pH} 7.4 \mathrm{PBS}$ for $24 \mathrm{~h}$ to ensure thorough protein internalization. To remove externally bound proteins in the present work, both AuNG-Lyz and AuNC-Lyz nanobioconjugates were subjected to a high-salt $(1 \mathrm{M} \mathrm{NaCl})$ buffer to screen the electrostatic attractions between nanoparticles and proteins. A solution comprised of $800 \mu l$ of nanobioconjugates in PBS mixed with $200 \mu \mathrm{l}, 5 \mathrm{M} \mathrm{NaCl}$ aqueous solution was shaken for $5 \mathrm{~min}$, making sure the nanobioconjugates were well dispersed from macro-aggregation. After shaking, nanobioconjugates were gently centrifuged at $5000 \mathrm{rpm}$ for $3 \mathrm{~min}$ twice to remove any unbound protein, and finally re-dispersed in $500 \mu$ of the $2 \mathrm{mM}$, pH 7.4 PBS. The centrifuged supernatants were subjected to a $\mu \mathrm{BCA}$ assay to measure the protein concentrations, and the protein amount remaining on AuNG or AuNC was calculated by subtracting the protein amount in the supernatant from the total added protein amount. A control group treated with DI water instead of $\mathrm{NaCl}$ solution was used for each nanobioconjugate titration to determine the amount of adsorbed proteins before $\mathrm{NaCl}$ wash. Moreover, according to our experiments, the presence of $1 \mathrm{M} \mathrm{NaCl}$ in $\mu \mathrm{BCA}$ had no observable effect on protein concentration measurements.

\subsection{Characterization of internally adsorbed proteins}

The enzymatic activity of Lyz-NP conjugates (either Lyz-AuNG or Lyz-AuNC) was determined spectrofluorometrically for the Lyz-catalyzed hydrolysis of 4-MU- $\beta$-(GluNAc) $)_{3}$ to yield 7-hydroxy-4-methylcoumarin (4-MU), and were also measured by a turbidity assay (M. lysodeikticu). The measurements followed existing protocols as described in detail by Qian et al.[12]. Protein conformation was analyzed via SERS. To prepare the Raman spectroscopy sample, 
AuNC-Lyz and NaCl-washed AuNG-Lyz nanobioconjugates were dropped onto a glass slide and covered by a microscope cover slip. The $785 \mathrm{~nm}$ wavelength laser was carefully focused between the slides via a 40X objective lens. To minimize the photon-induced alteration of the samples, the laser power was set at its lowest value ( $2 \mathrm{~mW}$ before the lens), the scanning time was $30 \mathrm{~s}$ and the final spectrum was accumulated in 5 scans. Scans were performed in both extended mode at 400 1800 $\mathrm{cm}^{-1}$, to obtain a general spectrum of the protein's Raman shift, and in static mode at $1100 \sim 1500 \mathrm{~cm}^{-1}$, to obtain the specific information of the Amide III band $\left(1200 \sim 1350 \mathrm{~cm}^{-1}\right)$. The baseline of the colloidal AuNG or AuNC solutions at the same measuring condition was subtracted from the spectra of the respective nano-bio samples. Lyz secondary structure was characterized using the analytical method reported by Cai and Singh[23]. Briefly, the spectra were deconvoluted and the areas under each of the deconvoluted peaks were calculated. The peaks around $1300 \mathrm{~cm}^{-1}$ were designated as $\alpha$-helix peaks, and their areas were compared with the summed areas of all the deconvoluted peaks, which gave the percentage of $\alpha$-helix in the Lyz secondary structure. The final secondary structural content was averaged from triple measurements. A $1 \mathrm{mM}$ free Lyz solution in the $2 \mathrm{mM}, \mathrm{pH}$ 7.4 PBS, with 20X stronger excitation intensity due to the absence of any Raman enhancement effect, was used as a control to examine the free protein conformation.

\section{Results and Discussion}

\subsection{Nanoparticles and surface ligands}

Typical SEM micrographs of AuNG and AuNC are shown in Figures 1A-D, exhibiting excellent nanoparticle uniformity in both size and morphology. The average size of the AuNG is 
$54 \pm 10 \mathrm{~nm}$, and AuNC is $74 \pm 14 \mathrm{~nm}$. The pores on the AuNG corners are $5 \sim 10 \mathrm{~nm}$ in diameter (Figure 1B), enabling access and protein internalization in the AuNG. The AuNG wall thickness varies from 5 10 nm, due to the curvature of the internal hollow. The 3-D structure of the AuNG's hollow core has been characterized by multiple advanced microscopic methods[13,24] and has been shown to be an irregular sphere or rounded cube. For example, Goris et al. characterized the 3-D morphology of such AuNGs via high-angle annular dark field-scanning transmission electron microscopy (HAADF-STEM)[13], as shown in Figure 1C. Quantitatively, our previous studies on protein-nanoparticle conjugates indicated that proteins (2 4 $\mathrm{nm}$ in size) are able to respond to the variation of surface curvature with radii over the range from $4 \sim 100 \mathrm{~nm}[7-9,17]$. The sizes of the internal hollow cores of the AuNGs are also within this scale range $(15 \sim 20 \mathrm{~nm}$ in radius), rendering them to be suitable platforms in this regard. The quantification of AuNG geometry followed the method developed by Qian et al.[12]. By combining the measured morphology and concentration data from SEM and ICP-MS, respectively, the nanoparticle concentrations of AuNC and AuNG were quantified, allowing reliable estimates to be made of protein uptake by AuNC and AuNG.

The nanoparticles (both AuNG and AuNC) underwent a ligand exchange process to replace their polyvinylpyrrolidone (PVP) surface ligands by 11-mercaptoundecanoic acid (11-MUA), which enhances protein affinity to the nanoparticles. According to SERS (Figure 1E), for the samples before ligand exchange, the peaks associated with the pyrrolidone groups $\left(856 \mathrm{~cm}^{-1}\right.$, ring breathing), methylene groups $\left(\mathrm{CH}_{2}\right.$ bending, $1400 \sim 1500 \mathrm{~cm}^{-1} ; \mathrm{CH}_{2}$ twisting, 1180-1300 $\mathrm{cm}^{-1}$; $\mathrm{CH}_{2}$ rocking, 720-1150 $\mathrm{cm}^{-1}$ ), and carbonyl groups (carbonyl stretching, $1583 \mathrm{~cm}^{-1}$ )[25,26], indicate that PVP polymer chains lay on the nanoparticle surface and are anchored via their carbonyl group, instead of dangling in solution[27]. On the other hand, the SERS of 11-MUA-capped AuNG has only three major peaks: at $713 \mathrm{~cm}^{-1}$, representing the vibration mode 
of the C-S bond in trans conformation; at $1100 \mathrm{~cm}^{-1}$, associated with the vibration mode of the C-C backbone; and the relatively weaker peak doublet at $1401 \mathrm{~cm}^{-1}$ and $1431 \mathrm{~cm}^{-1}$ that is attributed to the overlapping of the vibration mode of $\mathrm{COO}^{-1}$ in free solution and the stretching mode of $\mathrm{CH}_{2}$, respectively[28]. It is notable that for the 11-MUA capped AuNG, the C-S band is in trans instead of gauche conformation, and the stretching modes of the carboxylic group are much less pronounced, indicating that the molecule bonds to the surface via its thiol head group, with the carbonyl tail group dangling free in solution[29-31]. Theoretically, 11-MUA can bond to Au/Ag surfaces via either its thiol or carbonyl group; however, in this particular ligand exchange process, the thiol group is preferred to replace the carbonyl group of PVP[27,29,32], and the prolonged ligand exchange process renders a close packing of 11-MUA on the nanoparticle surface[33]. Since the dangling carboxylic groups are effectively deprotonated in $\mathrm{pH} 7.4$ buffer, the 11-MUA capped nanoparticle surfaces are thus charged and can ion-pair effectively with positively charged Lyz[34]. 


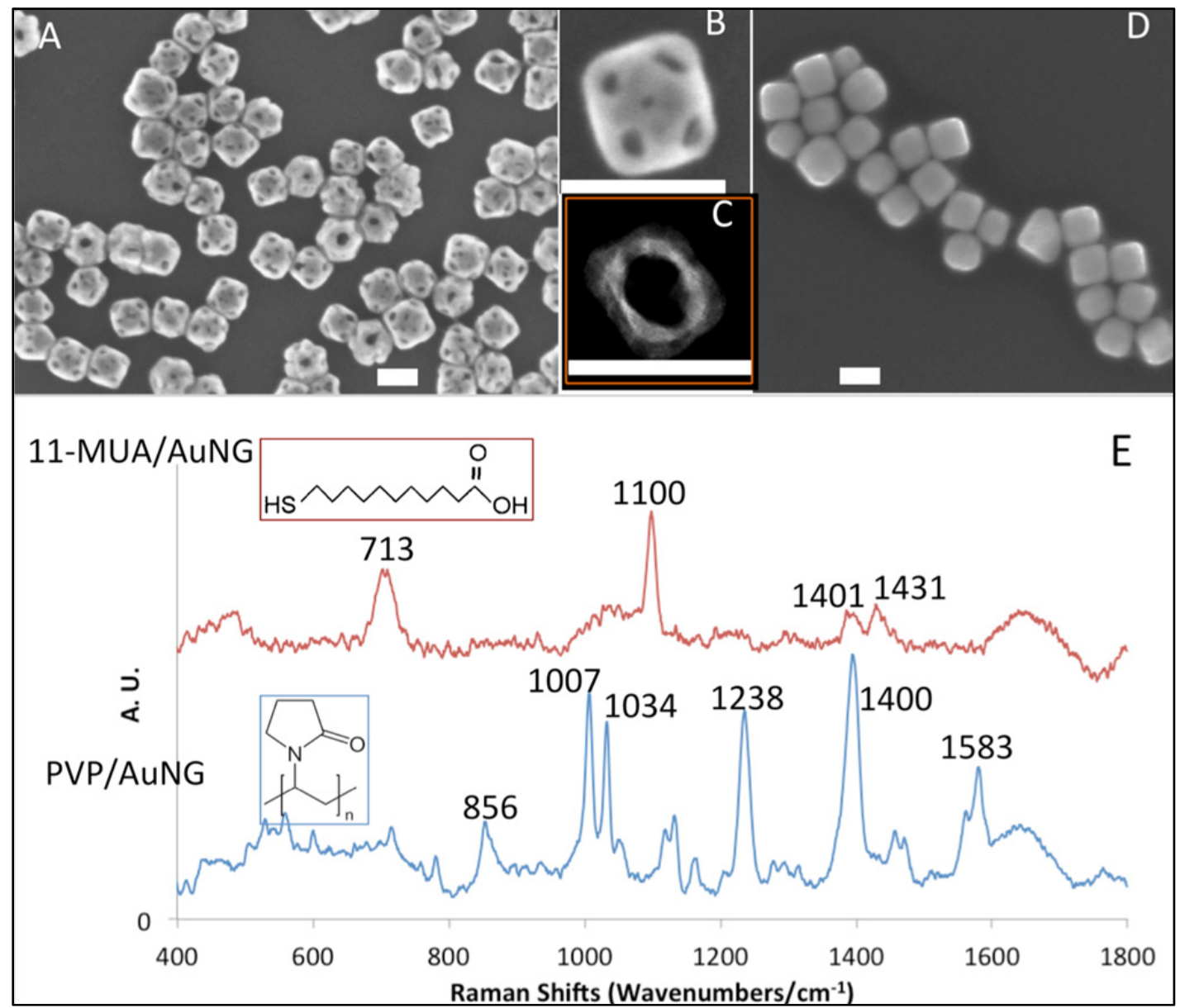

Figure 1. Characterization of nanoparticles: (A) SEM micrograph of AuNG; (B) closer visualization of one typical AuNG nanoparticle; (C) HAADF-STEM micrograph that exhibits the internal structure of AuNG[13]*; (D) SEM micrograph of AuNC; (E) Surface-enhanced Raman spectra (SERS) of AuNG before (bottom) and after (top) ligand exchange (top inset: 11-MUA; bottom inset: PVP). Scale bars $=50 \mathrm{~nm}$.

* Adapted with permission from Ref. 13. Copyright (C) 2014 American Chemical Society

\subsection{Selective removal of externally adsorbed proteins}

We have shown that 11-MUA capped AuNG can adsorb Lyz on both its internal and external surfaces, with internalization of Lyz taking place by free protein diffusion[12]. According to the previously established protein uptake profiles[12], it was observed that at least $40 \sim 50 \%$ of 
the adsorbed Lyz molecules were externally bound, depending on the free Lyz concentrations used. As Lyz molecules are bound to AuNG surfaces through electrostatic interactions, we reasoned that externally bound Lyz could be removed by eliminating such interactions. Two common methods for eliminating strong electrostatic attraction between Lyz $(\mathrm{pI} \sim 11)$ and 11-MUA include changing the solution $\mathrm{pH}$ to alter the net charges of AuNP and Lyz or increasing the solution ionic strength to shield the ionic interaction between 11-MUA and Lyz. To simplify the procedure and minimize possible damage to the adsorbed Lyz, we opted to use a high salt concentration $(1 \mathrm{M} \mathrm{NaCl})$ buffer to remove external Lyz, as shown schematically in Figure 2A.

The NaCl-washed nanobioconjugates were assayed for both the number of retained Lyz molecules and residual enzymatic activity. The assay methods used were described in detail by Qian et al.[12]. As shown in Figure 2C, after the high concentration $\mathrm{NaCl}$ incubation and subsequent wash, little Lyz remained on the AuNC surfaces $(<100 /$ AuNC), whereas $~ 3000$ Lyz were retained in the AuNG-Lyz, according to $\mu \mathrm{BCA}$ assay. In the fluorescence activity assay used, which detected both internally and externally absorbed Lyz using a small, highly diffusible substrate (4-MU- $\left.\beta-(\mathrm{GluNAc})_{3}\right)$, the high-salt washed AuNG-Lyz exhibited significant apparent activity, whereas the similarly washed AuNC-Lyz exhibited almost no activity. On the other hand, in the classical $M$. lysodeikticus cell turbidity activity assay also used, where the bacterial cells act as the substrate, neither AuNG-Lyz nor AuNC-Lyz exhibited detectable activity (data not shown). In our previous study[12] on non-washed Lyz-AuNG nanobioconjugates, we demonstrated that the cell turbidity assay can only measure externally-adsorbed Lyz on AuNG, while the fluorescence assay can measure both the external and internal Lyz, due to the different AuNG core accessibilities of the very different substrates in these two assays. Therefore, it was concluded in the present work that both AuNG's and AuNC's external surface proteins were desorbed by the high concentration $\mathrm{NaCl}$ wash, which is consistent with a previous report on high-salt 
desorption[35]. After re-dispersion in the $2 \mathrm{mM}, \mathrm{pH} 7.4 \mathrm{PBS}$, most of the remaining Lyz in the AuNG-Lyz nanobioconjugates was re-adsorbed at the internal surface, while the external Lyz on AuNG remained selectively removed by the high-salt wash.

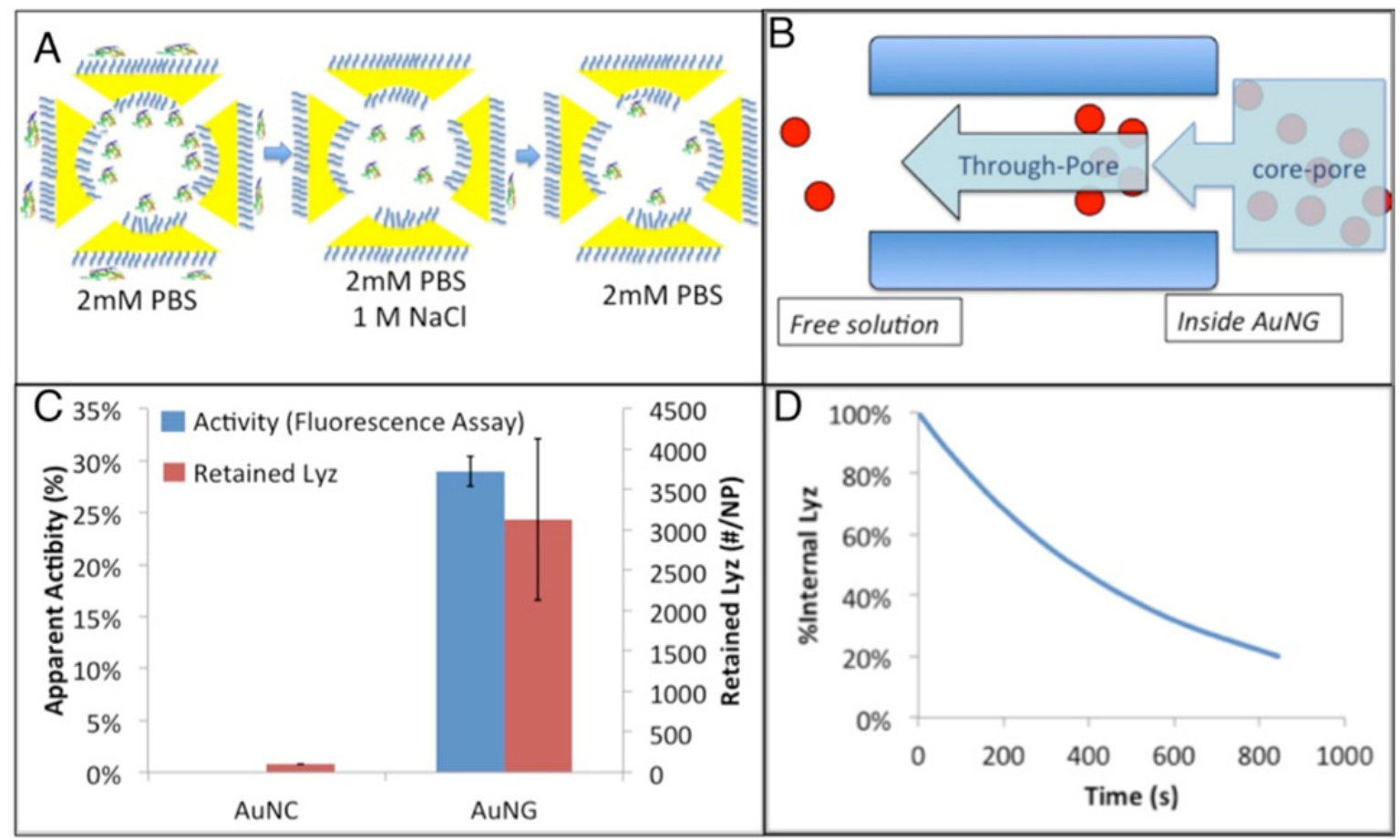

Figure 2. High-salt wash of nanobioconjugates: (A) Schematic illustration of protein removal in high-salt buffer; (B) Schematic illustration of Lyz (red spheres) diffusing out from AuNG through a pore (not drawn to scale); (C) Percentage of retained Lyz (red) and apparent activity (blue) on nanoparticles after 5 min $\mathrm{NaCl}$ wash, in which the $\mathrm{Lyz}$ was quantified by a $\mu \mathrm{BCA}$ assay, the value of $100 \%$ adsorbed proteins was determined from the protein uptake profile previously obtained[12], and the percentage activities were obtained by comparing the nanobioconjugate's fluorescence activity before and after the $\mathrm{NaCl}$ wash; (D) Calculated percentage of Lyz remaining in AuNG versus time.

To understand this phenomenon, we employed a model to quantify the protein desorption process. Note that while both internally and externally adsorbed Lyz are susceptible to desorption 
upon introduction of high ionic strength buffer, leaching of internal Lyz is inhibited due to the cage geometry. Small pores on the AuNG surfaces hinder through-pore diffusion when compared to bulk diffusion in solution, as depicted in Figure 2B. To simplify this mechanism, we assumed that all Lyz molecules are desorbed immediately after the addition of $1 \mathrm{M} \mathrm{NaCl}$. External Lyz molecules are simply released into the bulk solution. However, internal Lyz molecules must diffuse through the AuNG pores to be released into the bulk solution. The former process is rapid, while the latter is slowed by hindered through-pore diffusion. In this case, two parameters determine this leaching process: Lyz migration from the hollow core into the pore channel (core-pore diffusion); and Lyz migration though the pore channel into the surrounding solution (through-pore diffusion).

Core-pore diffusion is determined by the Lyz concentration difference between the pore and the hollow core, as described in Eq 1:

$$
J_{\text {core-pore }}=\left\{\begin{array}{c}
D_{0} \frac{C_{\text {core }}-C_{\text {pore }}}{\frac{d}{2}}, t=0 \\
D_{0} \frac{C_{\text {core }}-C_{\text {solution }}}{\frac{d}{2}}, t>0
\end{array}\right.
$$

where $J_{\text {core-pore }}$ denotes the protein flux into AuNG pores, $C_{\text {core }}$ denotes the protein concentration inside AuNG hollow cores, $C_{\text {pore }}$ denotes the protein concentration inside AuNG pores, and $\mathrm{D}_{0}$ is the protein diffusivity in free solution, calculated by the Stokes-Einstein equation.

Similarly, the through-pore diffusion is described by Eq 2:

$$
J_{\text {through-pore }}=\left\{\begin{array}{l}
D \frac{C_{\text {pore }}-C_{\text {solution }}}{l}, t=0 \\
D \frac{C_{\text {core }}-C_{\text {solution }}}{l}, t>0
\end{array}\right.
$$

where $J_{\text {thru-pore }}$ denotes the protein flux through the AuNG pores, $C_{\text {solution }}$ denotes the free protein concentration outside AuNG, and $D$ denotes the through-pore diffusivity calculated by the Higdon-Muldowney[36] equation (Eq 3), where $\lambda$ represents diffusant/pore diameter ratio: 


$$
\begin{aligned}
\frac{D}{D_{0}}= & 1+\frac{9}{8} \lambda \ln \lambda-1.5603 \lambda+0.528155 \lambda^{2}+1.91521 \lambda^{3}-2.81903 \lambda^{4}+0.270788 \lambda^{5}+ \\
& 1.10115 \lambda^{6}-0.435933 \lambda^{7}
\end{aligned}
$$

To quantify our approximation, the following representative dimensions were chosen: the hollow cores (assumed here to be spheres) are $40 \mathrm{~nm}$ in diameter; the cylindrical pores are $5 \mathrm{~nm}$ in radius and $10 \mathrm{~nm}$ in length; and the globular protein is $1.5 \mathrm{~nm}$ in radius. $\mathrm{C}_{\text {core }}(\mathrm{t}=0)=20 \mathrm{mM}$ is approximated according to the previously reported protein uptake profile[12], and $\mathrm{C}_{\text {solution }}(\mathrm{t}=0)=$ $20 \mu \mathrm{M}$ is considered as constant during protein leaching. These numerical values represent the typical experimental quantities used in this present study.

Because the pore surfaces are also covered by adsorbed protein molecules, a critical hypothetical boundary condition of significant hindered through-pore diffusion can be estimated at $t=0$. Quantitatively, the number of protein molecules inside each pore may be about $5-10$ (equilibrated with the hollow core), and due to the small pore volume and crowded pore environment, these crowding pore molecules can be approximately treated as one large diffusant molecule that gives an effective $\lambda$ of approx. 0.9 , resulting in $D \approx 10^{-4} D_{0}$ according to Eq (3). Using this $D$ value, along with the approximate quantities given above, Eqs (1) and (2) yield that $J_{\text {core-pore }}>J_{\text {thru-pore }}$ at $t=0$. This indicates not only that the through-pore diffusion is rate-determining at the beginning of the leaching process, but also that the pore would remain filled by diffusant until $C_{\text {core }}$ is at near equilibrium with $C_{\text {solution }}$. Therefore, $\lambda$ remains at around 0.9 and $D$ remains orders of magnitude lower than $D_{0}$ during most of the leaching process. From these estimates, it is apparent that even if the salt equilibrium time is neglected, $\sim 20 \%$ of the internal protein would still remain inside AuNG after 10 min of leaching (Figure 2D), explaining why AuNG can preserve almost half of its internally adsorbed Lyz after high-concentration $\mathrm{NaCl}$ incubation. 


\subsection{Selective characterization of internally adsorbed proteins: structure and activity}

The removal of externally adsorbed Lyz allowed us to selectively characterize the protein adsorbed onto the internal concave surfaces of AuNG. SERS was employed to characterize Lyz secondary structure. The amide III band $\left(1200 \mathrm{~cm}^{-1} \sim 1350 \mathrm{~cm}^{-1}, \mathrm{C}-\mathrm{N}\right.$ stretch and N-H bend) was selected as the window to observe changes to Lyz secondary structure, as each type of secondary structure has a relatively distinct peak region within this band[37]. Moreover, a rigorous spectral analytical method for the amide III band has been developed by Cai and Singh et al. [23,38,39], which further facilitated the quantitative analysis of the amide III band. AuNC-Lyz nanobioconjugates without high- $\mathrm{NaCl}$ wash were also used to assess Lyz behavior on the flat surfaces of nanocubes, as a reference for Lyz on concave surfaces. Figure 3A shows the measured Raman spectra between 400 2000 $\mathrm{cm}^{-1}$ of free Lyz, AuNC-Lyz, and AuNG-Lyz after Lyz removal from their external surface. Compared with free Lyz, clear red shifts were observed in the amide III band of AuNG-Lyz and AuNC-Lyz spectra, indicating that some Lyz domains were converted from $\alpha$-helices to $\beta$-sheets, $\beta$-strands or random coils. Moreover, the red shift of AuNG-Lyz was even more pronounced than that of AuNC-Lyz, indicating that concave surfaces may cause even greater structural perturbation than a nanoscale flat surface for these systems[40]. Using the static scan mode at amide III bands in Figure 3B, peak doublets were observed at $1250 \mathrm{~cm}^{-1}$ and 1300 $\mathrm{cm}^{-1}$. The peaks at $1300 \mathrm{~cm}^{-1}$ were assigned to $\alpha$-helices, and the broad peaks around $1250 \mathrm{~cm}^{-1}$ was assigned to the overlap of $\beta$-sheets $\left(\sim 1243 \mathrm{~cm}^{-1}\right)$ and random coils $\left(\sim 1265 \mathrm{~cm}^{-1}\right)[41]$. The $\alpha$-helix content of each sample was quantified, and an estimation of $\alpha$-helix content is given in Figure 3 C. The secondary structure of free Lyz consists of $\sim 44 \% \alpha$-helices[7,42], and the $\alpha$-helix contents of AuNC-Lyz and AuNG-Lyz were estimated to be $33 \%$ and $22 \%$, respectively. The structural analysis of native Lyz in the present study is consistent with the IR characterization result of Cai and Singh[23]; also, the Raman characterization of AuNC-Lyz's secondary structure 
in the present study is consistent with that of our previous investigation using circular dichroism[43].

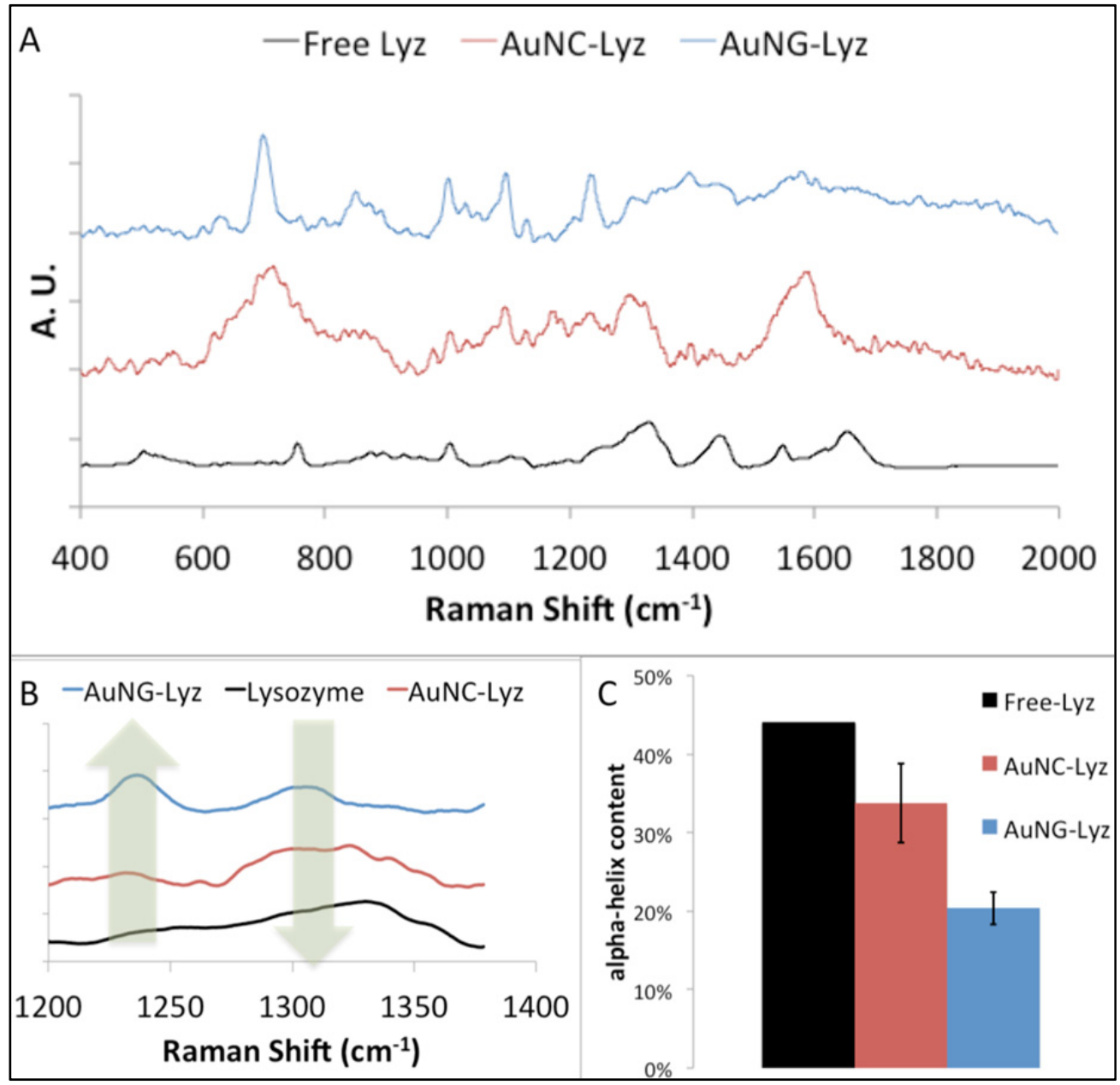

Figure 3. Raman or surface enhanced Raman spectroscopy (SERS) of free and immobilized Lyz: (A) SERS of AuNC-Lyz (red), AuNG-Lyz (blue) and Raman spectrum of free Lyz (black) in extended scanning mode $\left(400 \sim 2000 \mathrm{~cm}^{-1}\right)$; (B) Typical Amide III band of AuNG-Lyz (blue), AuNC-Lyz (red) and free Lyz (black), respectively, from a typical static mode scan; and (C) Relative $\alpha$-helix content of free Lyz, AuNC-Lyz and AuNG-Lyz, respectively.

Our results indicate that adsorbed Lyz undergoes a higher degree of denaturation on the concave inner surface of the AuNG than on the nanoscale flat surface of the AuNC. This is 
explained by the existing theory that as the contact area between the protein and adsorption surface increases, as with concave surfaces, so do interaction forces, resulting in more perturbation of the protein secondary structure[7,19,20,44]. As shown schematically in Figure 4, when protein molecules are densely packed on a nanoparticle surface, the surface contact area follows the sequence of convex (Figure 4A) $<$ flat (Figure 4B) $<$ concave (Figure 4C), and this roughly correlates to loss of the protein native structure and catalytic activity (in going from left to right in Figure 4). Quantitatively, if the protein radius is $1.5 \mathrm{~nm}$, the radii of surface curvature are $+10 \mathrm{~nm}$ (convex), $\infty$ (flat) and $-10 \mathrm{~nm}$ (concave), respectively, and if the interaction distance is $0.3 \mathrm{~nm}[44]$, the interaction volumes are $0.35 \mathrm{~nm}^{3}, 0.40 \mathrm{~nm}^{3}$ and $0.46 \mathrm{~nm}^{3}$ for the convex, flat and concave surfaces, respectively. The increased calculated interaction volumes, as determined in the present work, explain why Lyz undergoes greater structural perturbation on the internal concave surfaces of AuNG than it does on the flat surfaces of AuNC. Wang et al.[45] also reported the effect of surface curvature on the pKa of 11-MUA attached onto gold nanoparticle (AuNP) surfaces[45], and suggested that surface curvature may affect the distances between the head groups of 11-MUA, and thereby alter their $\mathrm{pKa}$ values. However, considering the actual radius in this study (flat vs. -10 $\mathrm{nm}$, which may change the head group distance by only $\sim 10 \%$ ), such a curvature effect would be minor when compared with the change of interaction volume. It is also worth noting that Leopold et al. reported ligand's pKa may be affected by surface smoothness[46]. Yet according to the microscopic studies on these nanoparticles[13,24,43], none of the investigated surfaces in this study are perfect, smooth surfaces, hence this mechanism could also be considered as minor. 
A

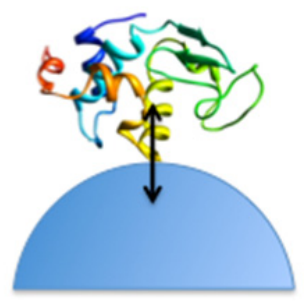

B

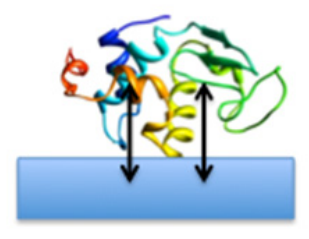

C

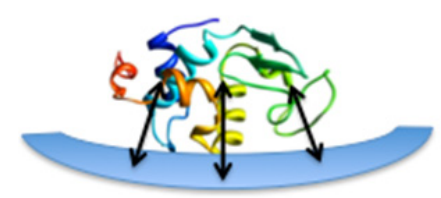

Figure 4. Schematic illustration of Lyz being adsorbed on: (A) convex; (B) flat; and (C) concave nanoscale surfaces. The black arrows represent electrostatic interactions between protein and surfaces.

The $\mathrm{NaCl}$ washed AuNG-Lyz were also assayed for their enzymatic activity in the fluorescence assay. By normalizing the apparent activity of $\mathrm{NaCl}$ washed AuNG-Lyz nanobioconjugates versus the retained Lyz amount, we obtained the specific enzymatic activity of Lyz on internal concave AuNG surfaces, which is approx. $60 \%$ of that of the free enzyme (Figure 5A). Thus, due to the structural perturbation induced by the concave surfaces, Lyz immobilized in AuNG has lower activity compared with that of native Lyz, which is consistent with the results of our previous studies on alkanethiol modified AuNP-Lyz nanobioconjugates[43,47]. Aggregation was observed in our AuNG-Lyz samples before and after $\mathrm{NaCl}$ wash, as indicated by the UV-vis spectra shown in Figure 5B, similar to observations of such aggregation in our previous AuNP-Lyz studies[43,47]. While it is not entirely clear why AuNP-Lyz tend to aggregate[48], a possible explanation is that a few adsorbed Lyz molecules may penetrate the 11-MUA self-assembled monolayer (SAM) and form an Au-S bond via the Cys residue with the gold surface, exposing Lyz's hydrophobic core and inducing aggregation[49]. It is worth noting that while AuNG-Lyz were subject to aggregation, they still remained considerably active compared with other aggregated AuNP-Lyz nanobioconjugates[43,47]. Of course, enzymatic activity is not only determined by protein structure, but is also determined by substrate accessibility[50,51]. In normal AuNP-Lyz nanobioconjugates, aggregation would significantly decrease the accessibility of 
substrates to Lyz trapped between nanoparticles (Figure 5D), while in AuNG-Lyz aggregates (Figure 5C), on the other hand, the Lyz immobilized inside AuNG would remain exposed to the solution, and thus remain accessible via the AuNG surface pores, leading to observed greater relative enzymatic activity.

Like convex surfaces, the protein structure-function relationship on concave surfaces could be very dependent on the radius of curvature. Some recent studies on mesoporous silica revealed that when proteins are closely confined in a hollow space (i.e., the dimension of the hollow space and protein are similar) they may display superactivity, despite showing some degree of structural perturbation[16,42]. These discoveries indicate that at concave surfaces, as the contact area between the protein and surface increases, and immobilized proteins are subjected to greater spatial confinement, nano-bio interactions can be significantly different from those on a convex or flat surfaces[5,48]. Beyond the current studies, more advanced spectroscopic characterizations may provide more information for adsorbed protein conformations, and thereby shed more lights on nano-bio interactions at concave surfaces. Other than the actual nanoparticle surface and protein, the surface ligands are affected more by surface curvature at this level[45], which further complicates the analysis. Hence, further research on the effect of concave surfaces with varied curvatures remains needed to provide comprehensive information of the effect of concave curvature on protein structure and function, particularly as the relative size between the surface and adsorbed proteins become similar. Due to their readily tunable morphology, AuNGs can serve as a useful nanoscale material for such studies. 


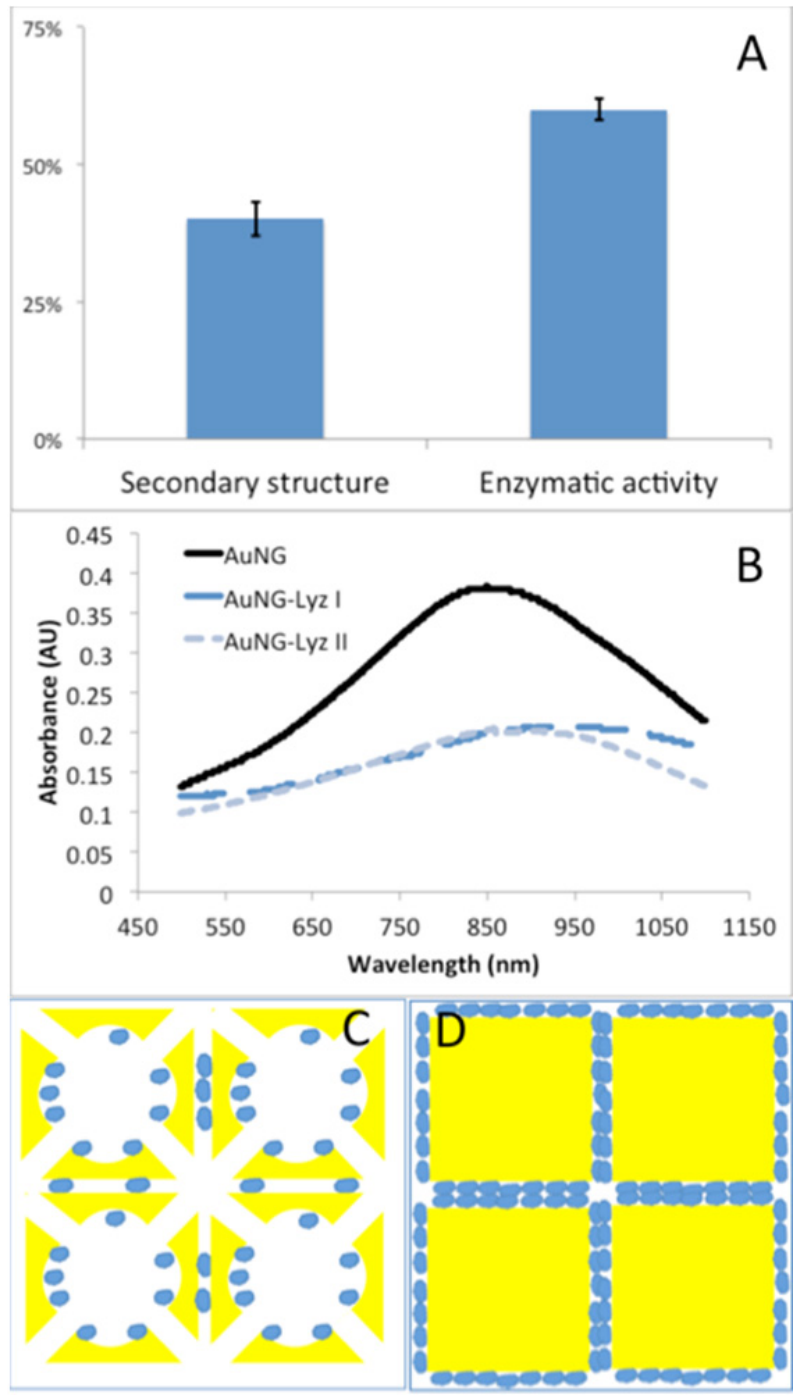

Figure 5 (A) Lyz behavior on AuNG concave surfaces, where "Secondary structure" denotes the percentage of $\alpha$-helices retained compared with native Lyz and "Enzymatic activity" denotes the percentage of retained activity of immobilized Lyz in AuNG conjugates compared to that of native Lyz; (B) UV-vis spectroscopy of 11-MUA capped AuNG nanoparticles (AuNG, black line), AuNG-Lyz before NaCl wash (AuNG-Lyz I, dark blue dash line) and AnNG-Lyz after NaCl wash (AuNG-Lyz II, light blue short dash line), respectively; (C) Schematic illustration of Lyz-AuNG nanobioconjugates after high-NaCl wash; (D) Schematic illustration of Lyz-AuNC aggregate without $\mathrm{NaCl}$ wash. The schematics are not to scale. 


\section{Conclusions}

Using specifically surface modified AuNGs in a high ionic strength buffer, we have been able to selectively remove the externally bound Lyz from AuNG-Lyz nanobioconjugates and take advantage of the hindered diffusion of Lyz through AuNG pores to restrain the internally adsorbed Lyz from leaching. As a result, nanobioconjugates with predominantly protein-concave surface interactions were obtained that could be selectively characterized. Subsequent SERS measurements and enzymatic activity assays revealed that the Lyz immobilized on the concave internal surfaces of the AuNG exhibited significant secondary conformational perturbation, yet remained substantially active. This study has thus provided unique insight into nano-bio interactions at concave surfaces and has, furthermore, introduced a simple new method to create nanoscale platforms that overcome the major methodological difficulties in the study of concave surface nano-bio interactions. By combining this platform with more advanced spectroscopic technologies, more detailed protein structural information could be resolved, and hence more information of this type of nano-bio interaction can be revealed. Finally, considering the ability to tailor the pore sizes and surface moieties of AuNG, combining with the reported versatile functionality of AuNP in protein adsorptions[10,11], we can envision considerable potential for these nano-bio systems as nanoscale therapeutic delivery vehicles.

\section{Acknowledgement}

This work was supported by the Nanoscale Science and Engineering Initiative of the National Science Foundation under Grant DMR-0642573. 


\section{References}

[1] Moon GD, Choi S-W, Cai X, Li W, Cho EC, Jeong U, et al. A New Theranostic System Based on Gold Nanocages and Phase-Change Materials with Unique Features for Photoacoustic Imaging and Controlled Release. J Am Chem Soc 2011;133:4762-5.

[2] Grover N, Dinu CZ, Kane RS, Dordick JS. Enzyme-Based Formulations for Decontamination: Current State and Perspectives. Appl Microbiol Biotechnol 2013;97:3293-300.

[3] Stanley SA, Gagner JE, Damanpour S, Yoshida M, Dordick JS, Friedman JM. Radio-wave heating of iron oxide nanoparticles can regulate plasma glucose in mice. Science Signalling 2012;336:604.

[4] Stanley SA, Sauer J, Kane RS, Dordick JS, Friedman JM. Remote regulation of glucose homeostasis in mice using genetically encoded nanoparticles. Nat Med 2014;21:92-8.

[5] Nuffer JH, Siegel RW. Nanostructure-Biomolecule Interactions: Implications for Tissue Regeneration and Nanomedicine. Tissue Eng 2009;16:423-30.

[6] Nel AE, Mädler L, Velegol D, Xia T, Hoek EMV, Somasundaran P, et al. Understanding Biophysicochemical Interactions at The Nano-Bio Interface. Nat Mater 2009;8:543-57.

[7] Vertegel AA, Siegel RW, Dordick JS. Silica Nanoparticle Size Influences The Structure and Enzymatic Activity of Adsorbed Lysozyme. Langmuir 2004;20:6800-7.

[8] Shang W, Nuffer JH, Dordick JS, Siegel RW. Unfolding of Ribonuclease A on Silica Nanoparticle Surfaces. Nano Lett 2007;7:1991-5.

[9] Shang W, Nuffer JH, Muniz-Papandrea VA, Colón W, Siegel RW, Dordick JS.

Cytochrome c on Silica Nanoparticles: Influence of Nanoparticle Size on Protein Structure, Stability, and Activity. Small 2009;5:470-6.

[10] Sisco PN, Wilson CG, Chernak D, Clark JC, Grzincic EM, Ako-Asare K, et al. Adsorption of Cellular Proteins to Polyelectrolyte-Functionalized Gold Nanorods: A Mechanism for Nanoparticle Regulation of Cell Phenotype? PLoS ONE 2014;9:e86670.

[11] Mahmoudi M, Lohse SE, Murphy CJ, Fathizadeh A, Montazeri A, Suslick KS. Variation of Protein Corona Composition of Gold Nanoparticles Following Plasmonic Heating. Nano Lett 2014;14:6-12. 
[12] Qian X, Levenstein A, Gagner JE, Dordick JS, Siegel RW. Protein Immobilization in Hollow Nanostructures and Investigation of the Adsorbed Protein Behavior. Langmuir 2014;30:1295-303.

[13] Goris B, Polavarapu L, Bals S, Van Tendeloo G, Liz-Marzán LM. Monitoring Galvanic Replacement Through Three-Dimensional Morphological and Chemical Mapping. Nano Lett 2014;14:3220-6.

[14] Khlebtsov N, Dykman L. Biodistribution and toxicity of engineered gold nanoparticles: a review of in vitro and in vivo studies. Chem Soc Rev 2011;40:1647.

[15] Mahmoud MA, Snyder B, El-Sayed MA. Surface Plasmon Fields and Coupling in the Hollow Gold Nanoparticles and Surface-Enhanced Raman Spectroscopy. Theory and Experiment. J Phys Chem C 2010;114:7436-43.

[16] Kao K-C, Lin T-S, Mou C-Y. Enhanced Activity and Stability of Lysozyme by Immobilization in the Matching Nanochannels of Mesoporous Silica Nanoparticles. J Phys Chem C 2014;118:6734-43.

[17] Shrivastava S, Nuffer JH, Siegel RW, Dordick JS. Position-Specific Chemical Modification and Quantitative Proteomics Disclose Protein Orientation Adsorbed on Silica Nanoparticles. Nano Lett 2012;12:1583-7.

[18] Shrivastava S, McCallum SA, Nuffer JH, Qian X, Siegel RW, Dordick JS. Identifying Specific Protein Residues That Guide Surface Interactions and Orientation on Silica Nanoparticles. Langmuir 2013;29:10841-9.

[19] Billsten P, Wahlgren M, Arnebrant T, McGuire J. Structural changes of T4 lysozyme upon adsorption to silica nanoparticles measured by circular dichroism. J Colloid Interf Sci 1995.

[20] Tian M, Lee WK, Bothwell MK, McGuire J. Structural stability effects on adsorption of bacteriophage T4 lysozyme to colloidal silica. J Colloid Interf Sci 1998.

[21] Skrabalak SE, Au L, Li X, Xia Y. Facile Synthesis Of Ag Nanocubes And Au Nanocages. Nat Protoc 2007;2:2182-90.

[22] Seo D, Park JC, Song H. Polyhedral Gold Nanocrystals with $\mathrm{O}_{\mathrm{h}}$ Symmetry: From Octahedra to Cubes. J Am Chem Soc 2006;128:14863-70. 
[23] Cai S, Singh BR. A Distinct Utility of the Amide III Infrared Band for Secondary Structure Estimation of Aqueous Protein Solutions Using Partial Least Squares Methods. Biochemistry 2004;43:2541-9.

[24] Takahashi Y, Zettsu N, Nishino Y, Tsutsumi R, Matsubara E, Ishikawa T, et al. Three-Dimensional Electron Density Mapping of Shape-Controlled Nanoparticle by Focused Hard X-ray Diffraction Microscopy. Nano Lett 2010;10:1922-6.

[25] Borodko Y, Habas SE, Koebel M, Yang P, Frei H, Somorjai GA. Probing the Interaction of Poly (Vinylpyrrolidone) with Platinum Nanocrystals by UV-Raman And FTIR. J Phys Chem B 2006;110:23052-9.

[26] Lepp A, Siiman O. Surface Resonance Raman and Surface-Enhanced Raman Scattering Excitation Profiles for Dabsyl Aspartate on Colloidal Silver in Ethanol. J Phys Chem 1985;89:3494-502.

[27] Wang H, Qiao X, Chen J, Wang X, Ding S. Mechanisms of PVP in the Preparation of Silver Nanoparticles. Mater Chem Phys 2005;94:449-53.

[28] Castro JL, Lopez-Ramirez MR, Arenas JF, Otero JC. Surface-Enhanced Raman Scattering of 3-Mercaptopropionic Acid Adsorbed on a Colloidal Silver Surface. J Raman Spectrosc 2004;35:997-1000.

[29] Laaksonen T, Ahonen P, Johans C, Kontturi K. Stability and Electrostatics of Mercaptoundecanoic Acid-Capped Gold Nanoparticles with Varying Counterion Size. ChemPhysChem 2006; 7:2143-9.

[30] Moskovits M, Suh JS. Conformation of Mono-And Dicarboxylic Acids Adsorbed on Silver Surfaces. J Am Chem Soc 1985;107:6826-9.

[31] Bryant MA, Pemberton JE. Surface Raman Scattering of Self-Assembled Monolayers Formed from 1-Alkanethiols at Ag. J Am Chem Soc 1991;113:3629-37.

[32] Zelikin AN, Such GK, Postma A, Caruso F. Poly(vinylpyrrolidone) for Bioconjugation and Surface Ligand Immobilization. Biomacromolecules 2007;8:2950-3.

[33] Ma C, Harris JM. Surface-Enhanced Raman Scattering Study of the Kinetics of Self-Assembly of Carboxylate-Terminated n-Alkanethiols on Silver. Langmuir 2012;28:2628-36. 
[34] Kuehner DE, Engmann J, Fergg F, Wernick M, Blanch HW, Prausnitz JM. Lysozyme Net Charge and Ion Binding in Concentrated Aqueous Electrolyte Solutions. J Phys Chem B 1999;103:1368-74.

[35] Liao M-H, Chen D-H. Fast And Efficient Adsorption/Desorption Of Protein By A Novel Magnetic Nano-Adsorbent. Biotechnology Letters 2002;24:1913-7.

[36] Lu S, Song Z, He J. Diffusion-Controlled Protein Adsorption in Mesoporous Silica. J Phys Chem B 2011;115:7744-50.

[37] Anderle G, Mendelsohn R. Thermal denaturation of globular proteins. Fourier transform-infrared studies of the amide III spectral region. Biophysical Journal 1987.

[38] Cai S, Singh BR. Identification of $\beta$-turn and random coil amide III infrared bands for secondary structure estimation of proteins. Biophysic Chem 1999:1-14.

[39] Singh BR, DeOliveira DB, Fu F-N, Fuller MP. Fourier transform infrared analysis of amide III bands of proteins for the secondary structure estimation. In: Nafie LA, Mantsch HH, editors. OE/LASE'93: Optics, Electro-Optics, \& Laser Applications in Science\& Engineering, vol. 1890, SPIE; 2004, pp. 47-55.

[40] Anderle G, Mendelsohn R. Thermal Denaturation of Globular Proteins: Fourier Transform-Infrared Studies of the Amide III Spectral Regions. Biophysical Journal 1987;52:69-74.

[41] Brunner H, Holz M, Jering H. Raman Studies on Native, Reduced, and Modified Basic Pancreatic Trypsin Inhibitor. Eur J Biochem 1974;50:129-33.

[42] Sang L-C, Coppens M-O. Effects of Surface Curvature and Surface Chemistry on the Structure and Activity of Proteins Adsorbed in Nanopores. Phys Chem Chem Phys 2011;13:6689-98.

[43] Gagner JE, Qian X, Lopez MM, Dordick JS, Siegel RW. Effect of Gold Nanoparticle Structure on the Conformation and Function of Adsorbed Proteins. Biomaterials 2012;33:8503-16.

[44] Lundqvist M, Sethson I, Jonsson B-H. Protein Adsorption onto Silica Nanoparticles: Conformational Changes Depend on the Particles' Curvature and the Protein Stability. Langmuir 2004;20:10639-47. 
[45] Wang D, Nap RJ, Lagzi I, Kowalczyk B, Han S, Grzybowski BA, et al. How and Why Nanoparticle's Curvature Regulates the Apparent pKa of the Coating Ligands. J Am Chem Soc 2011;133:2192-7.

[46] Leopold MC, Black JA, Bowden EF. Influence of Gold Topography on Carboxylic Acid Terminated Self-Assembled Monolayers. Langmuir 2002;18:978-80.

[47] Gagner JE, Lopez MD, Dordick JS, Siegel RW. Effect of Gold Nanoparticle Morphology on Adsorbed Protein Structure and Function. Biomaterials 2011;32:7241-52.

[48] Gagner JE, Shrivastava S, Qian X, Dordick JS, Siegel RW. Engineering Nanomaterials for Biomedical Applications Requires Understanding the Nano-Bio Interface: A Perspective. J Phys Chem Lett 2012;3:3149-58.

[49] Zhang D, Neumann O, Wang H, Yuwono VM, Barhoumi A, Perham M, et al. Gold Nanoparticles Can Induce the Formation of Protein-based Aggregates at Physiological pH. Nano Lett 2009;9:666-71.

[50] Ding H-M, Shao L, Liu R-J, Xiao Q-G, Chen J-F. Silica nanotubes for lysozyme immobilization. J Colloid Interf Sci 2005;290:102-6.

[51] Rodrigues RC, Ortiz C, Berenguer-Murcia Á, Torres R, Fernández-Lafuente R. Modifying enzyme activity and selectivity by immobilization. Chem Soc Rev 2013;42:6290. 